\title{
Correlation Analysis of Chitosan Physicochemical Parameters Determined by Different Methods
}

\author{
Tatiana Lyalina ${ }^{1}$, Anastasia Zubareva ${ }^{1 *}$, Sergey Lopatin ${ }^{1}$, Vitaly Zubov ${ }^{2}$, Svetlana Sizova ${ }^{2}$, ${ }^{*}$ Elena \\ Svirshchevskaya ${ }^{2}$ \\ ${ }^{1}$ Institute of Bioengineering, Research Center of Biotechnology of the Russian Academy of Sciences, Russia \\ ${ }^{2}$ Shemyakin-Ovchinnikov Institute of Bioorganic Chemistry of the Russian Academy of Sciences, Russia
}

Submission: November 17, 2016; Published: January 03, 2017

"Corresponding author: Elena Svirshchevskaya, Institute of Bioorganic Chemistry of the Russian Academy of Sciences, Russia

\begin{abstract}
Chitosan is a natural biodegradable cationic polymer with a promising potency for different applications. Its use is limited by standardization problems related to chitosan nature. The aim of this work was to compare different methods of molecular weight (MW) and deacetylation degree (DD) determination using two chitosan series varied in MW and DD. The absolute DD appeared to be accurately determined by NMR, first derivative of UV-absorbance spectra analysis, or conductometric titration. Contrary to DD, MW estimated by viscosity, size exclusion chromatography, or asymmetric field flow fractionation did not provide comparable absolute MW values. At the same time, correlation between all the methods was extremely high (Pearson's coefficient of correlation, $\mathrm{r} \sim 1$ ) showing that each of them is sensitive to the change in chitosan MW. Taken collectively it can be concluded that chitosan behaves in different conditions as a molecule with different MW. As MW determined by any of these methods and viscosity correlate highly significantly, viscosity as a directly determined value is recommended to be used as the main chitosan characteristic.
\end{abstract}

Keywords: Chitosan; Molecular weight; Deacetylation degree; Asymmetric field flow fractionation; size exclusion chromatography; Viscometry

\section{Introduction}

Deacetylated chitin derivative chitosan is an attractive biopolymer due to its biocompatibility, biodegradability, low toxicity, and abundant reactive groups. Nevertheless practical application of chitosan and its derivatives is limited by standardization problems. These difficulties are caused by the nature of chitosan (variety of sources, dispersity, impurities) as well as the variations in molecular weight (MW) and acetylation degree (DD) determined by different methods. In 2011 German company Heppe Medical Chitosan GmbH sent chitosan samples to eleven European laboratories in order to independently determinate MW and DD. The data on DD were consistent while MW of the same sample varied in a broad range [1] resulted in the conclusion about intrinsic problems of chitosan standardization.

DD can be determined by three different types of methods: spectral (1H-NMR, 13C-NMR, 15N-NMR, UV, IR); analytical (conductometric and potentiometric titration, ninhydrin method); and degradation (elemental analysis, complete hydrolysis of chitosan followed by colorimetric or HPLC analysis, differential thermal analysis) [2]. There are no published papers which compared all these methods, however a few of them were compared by many groups [3-5].

Determination of chitosan MW, in our opinion, is a more complicated problem. This relates to the dispersity of the polymer and its tendency to self-association in solutions [6,7]. A variety of methods currently used to estimate chitosan MW (size exclusion chromatography (SEC), SEC coupled with MALS detector, viscometry, osmometry, dynamic and static light scattering, end group analyses, analytical ultracentifugation, et cetera) produce results which are often poorly compatible $[8,9]$.

The problem of dispersity can be partially solved by fractionation methods, such as ones developed by Giddings $[10,11]$ and nowadays realized by Post nova Analytics company (Germany). This group of methods is based on the separation of macromolecules as well as nanoparticles in a flow of liquid under the influence of perpendicular acting forces of different nature (sedimentation or thermal field, perpendicular flow etc.)[12]. In this paper a comparative study and correlation analysis of DD and MW, obtained by different methods including asymmetric field flow fractionation, were carried out. 


\section{Experimental}

\section{Materials}

Table 1: Viscometry protocols and Mark-Houwink empirical coefficients.

\begin{tabular}{|c|c|c|c|c|c|c|c|}
\hline & & \multicolumn{2}{|c|}{ Protocol 1[17] } & \multicolumn{2}{|c|}{ Protocol 2 [19] } & \multicolumn{2}{|c|}{ Protocol 3 [18] } \\
\hline \multicolumn{2}{|c|}{ Buffer $\mathrm{AcOH} / \mathrm{AcONa}$} & \multicolumn{2}{|c|}{$0.2 \mathrm{M} / 0.1 \mathrm{M}$} & \multicolumn{2}{|c|}{$0.2 \mathrm{M} / 0.2 \mathrm{M}$} & \multicolumn{2}{|c|}{$0.3 \mathrm{M} / 0.2 \mathrm{M}$} \\
\hline \multicolumn{2}{|c|}{ Temperature, ${ }^{\circ} \mathrm{C}$} & \multicolumn{2}{|c|}{30} & \multicolumn{2}{|c|}{25} & \multicolumn{2}{|c|}{25} \\
\hline \multicolumn{2}{|c|}{ Methods $^{a}$} & \multicolumn{2}{|c|}{ Light Scattering } & \multicolumn{2}{|c|}{ Sedimentation and Diffusion } & \multicolumn{2}{|c|}{ Multidetector SEC } \\
\hline \multirow{2}{*}{ Sample } & \multirow{2}{*}{ DD } & \multicolumn{6}{|c|}{ Mark-Houwink coefficients } \\
\hline & & $\mathrm{k}^{\mathrm{b}}$ & $\alpha^{\mathrm{b}}$ & $\mathrm{k}^{\mathrm{c}}$ & $\alpha^{\mathrm{c}}$ & $\mathrm{k}^{\mathrm{d}}$ & $\alpha^{\mathrm{d}}$ \\
\hline A1 & 90 & 0.00375 & 0.902 & \multirow{5}{*}{0.00014} & \multirow{5}{*}{0.85} & \multirow{5}{*}{0.076} & \multirow{5}{*}{0.76} \\
\hline A2 & 68 & $7.4 \mathrm{E}-05$ & 1.126 & & & & \\
\hline A3 & 56 & $5 \mathrm{E}-06$ & 1.248 & & & & \\
\hline B & 90 & 0.00375 & 0.902 & & & & \\
\hline $\mathrm{C}$ & 92 & 0.0051 & 0.882 & & & & \\
\hline
\end{tabular}

a Methods used to determine Mark-Houwink coefficients in papers [17-19].

b-d k and $\alpha$-empirical Mark-Houwink coefficients according to each protocol.

${ }^{\mathrm{e}} \mathrm{DD}$ was calculated using equation 1.

Protocol 1 was proposed for chitosan with different DD; protocols 2 and 3 describe the analysis

of chitosan with high DD.

Chitosan (A1, $200 \mathrm{kDa}$, DD 90\%) (Table 1) and chitosan $1000 \mathrm{kDa}$, DD 87\% were purchased from ZAO «Bioprogress», Russia. Chitosan $1000 \mathrm{kDa}$ was used to obtain samples B and C (Table 1) by acidic hydrolysis. Glacial acetic acid (AcOH), sodium hydroxide $(\mathrm{NaOH})$, hydrochloric acid $(\mathrm{HCl})$, acetic anhydride (Ac20), ammonium hydroxide $\left(\mathrm{NH}_{4} \mathrm{OH}\right)$ (reagent-grade), methanol (MeOH) (Sigma-Aldrich, USA) were used as received.

\section{Physicochemical characterization of chitosan}

Chitosan was purified as described earlier [13]. To obtain chitosan samples with different DD and the same MW, A1 was re-acetylated as described earlier [14]. Chitosan with MW 50 $\mathrm{kDa}$ (sample B) and $\sim 20 \mathrm{kDa}$ (sample C) were prepared by acidic hydrolysis of chitosan $1000 \mathrm{kDa}$ as described [15].

a) Conductometric titration: Analytical sample (0.1g) of chitosan was dried to a constant weight in microwave oven. Standard $0.1 \mathrm{MHCl}$ solution was prepared using standard titrimetric substance. Concentration of $\mathrm{NaOH}$ solution was evaluated with standard $0.1 \mathrm{M} \mathrm{HCl}$ solution. Sample was dissolved in $10 \mathrm{ml} 0.1 \mathrm{M} \mathrm{HCl}$ and then diluted with $40 \mathrm{ml}$ of distilled H2O. For titration curve plotting every $30 \mathrm{sec} 100 \mu \mathrm{l}$ of $\mathrm{NaOH}$ titrated solution were added. b) Proton nuclear magnetic resonance (1H-NMR): Proton spectra of chitosan and its derivatives were registered by Avance III (Bruker, Germany) spectrometer (operating frequency for the protons- $600 \mathrm{MHz}$, temperature $30{ }^{\circ} \mathrm{C}$ ). The samples were prepared in D20 with addition of DCl until complete chitosan dissolution. Signals of the solvent were suppressed by selective impulses using gradient.

c) First derivative ultraviolet spectrophotometry (FDUV): Analysis was carried out according to the method described earlier [16] using Shimadzu UV-1601PC spectrophotometer. For the exclusion of the solvent influence, UV-spectra of $\mathrm{AcOH}$ at different concentrations $(0.01 \mathrm{M}, 0.02 \mathrm{M}, 0.03 \mathrm{M})$ were recorded and the crossing point (zero crossing point, ZCP) was found $(203 \mathrm{~nm})$. Calibration curve was plotted using $\mathrm{N}$-acetyl-D-glucosamine in AcOH UV spectra. Distance from the ZCP to the spectrum (in optical density units, $\Delta \mathrm{D}$, O.D.) was measured.

d) Asymmetric flow field fractionation: Fractionation of polymers was carried out using AF 2000 MF Flow FFF System (AF4) (Post nova Analytics, Germany) with following parameters: channel thickness $350 \mu \mathrm{m}$, PES separating membrane with $10 \mathrm{kDa}$ cutoff limit, flow speed $0.3 \mathrm{ml} / \mathrm{min}$. Chitosan samples 0.1-0.5\% (w/v) in 0.2 M AcOH/AcONa buffer $(\mathrm{pH}=4.5)$ were prepared for the analysis. Characteristics of each fraction were studied using refractometric $(\mathrm{dn} / \mathrm{dc}$ value was $0.181 \mathrm{ml} / \mathrm{g}$ ) and multi-angle light scattering (MALS) detectors.

e) Dynamic viscosity studies: Determination of dynamic viscosity $\left(\eta_{d}\right)$ was implemented using Fungi lab Smart $\mathrm{L}$ rotation viscometer under with following conditions: 


\section{Organic and Medicinal Chemistry International Journal}

temperature $25^{\circ} \mathrm{C}$, rotation speed $100 \mathrm{rpm}$, spindle $\mathrm{L} 2$. Chitosan $1 \%(\mathrm{w} / \mathrm{v})$ in $0.2 \mathrm{M} \mathrm{AcOH} / 0.1 \mathrm{M}$ AcONa buffer $(\mathrm{pH}=4.4)$ was prepared for the measurement. Dynamic viscosity values (mPa.s) were calculated as an average of three independent measurements.

f) Viscometry: Viscometry was conducted using three published protocols (Table 1). Chitosan viscosity average molecular weights $\left({ }^{\overline{M_{n}}}\right)$ was calculated according to MarkHouwink equation using published empirical coefficients (Table 1) [17-19].

g) Size exclusion chromatography: Number average $\left(\overline{M_{n}}\right)$, weight average $\left(\overline{M_{w}}\right)$, and z-average $\left(\overline{M_{z}}\right)$ MW were determined by high-performance size exclusion chromatography (SEC) using S2100 (Sykam, Germany) chromatograph, Ultahydrogel-250 $(7.8 \times 300 \mathrm{~mm})$ (Waters, USA) column, GFC-4000 $(4 \times 3 \mathrm{~mm})$ (Phenomenex, USA) precolumn. Elution was carried out with $0.05 \mathrm{M} \mathrm{AcOH}, 0.15 \mathrm{M}$ $\mathrm{AcNH}_{4}$ solution $(\mathrm{pH}=5.1)$ at flow $0.5 \mathrm{ml} / \mathrm{min}$ and temperature $30^{\circ} \mathrm{C}$. Separation process was controlled using refractometric detector K-2301 (Knauer, Germany). Molecular-weight distribution values were calculated with "Multichrom" 1.6 software ("Ampersend", Moscow, Russia). Different MW dextrans for SEC (Sigma, USA) were used to calibrate the column.

\section{Theory}

a) Estimation of DD using $1 \mathrm{H}-\mathrm{NMR}$ spectra: DD was calculated from 1H-NMR spectra by two equations most often used. In the first case DD was obtained by calculating the ratio between proton integrals at 3.2 and $2.0 \mathrm{ppm}$ (Figure 1) using equation 1.

$\left.\mathrm{DD}=\left(3 \mathrm{~A}_{H 2-G l c N}\right) / \mathrm{A}_{C H 3}+3 \mathrm{~A}_{H 2-G l C N}\right) .100 \%$,

where $A_{x}$ is an integral of proton $X$ signal.

As an alternative, the equation taking into account the ratio of integrals of all glucopyranose protons signals (Figure 1) marks 3 and 1 to the protons of acetyl group of NAcGlcN (Figure 1) was chosen equation 2 .

$$
\mathrm{DD}=100-\left(2 \mathrm{~A}_{\mathrm{CH} 3} / \mathrm{A}_{\Sigma H 2-H 6}\right) \cdot 100 \%,
$$

b) Estimation of DD using conductometric titration curves: There are also two methods to calculate DD on the basis of the titration curves. The first one uses only part 1 of the curve (Figure 1 b) equations 3,4 :

$$
\begin{gathered}
x=\mathrm{N} 1 . \mathrm{V} 1-\mathrm{N} 2 . \mathrm{V} 2 \\
\mathrm{DD}=\mathrm{x} /[\mathrm{x}+(\mathrm{m} .0 .9-\mathrm{x} .161) / 203] .100 \%,
\end{gathered}
$$

where $\mathrm{V} 1$ is volume of acid solution, $\mathrm{ml}$; V2 is volume of alkaline solution required for the titration, $\mathrm{ml}$; $\mathrm{N} 1$ is normal concentration of acid solution, Eq/ml; $\mathrm{N} 2$ is normal concentration of alcaline solution, Eq/ml; 161 and $203 \mathrm{~g} / \mathrm{mol}$ are the molar weights of GlcN and NAcGlcN units accordingly; $m$ is the mass of the sample, $\mathrm{g}$. The second one takes into account the difference between volumes of $\mathrm{NaOH}$ used for the neutralization of unbound $\mathrm{HCl}$ and consumed to titer free amino groups in the polymer chain equation 5 :

$$
\mathrm{DD}=\mathrm{C}_{\mathrm{NaOH}}(\mathrm{V} 2-\mathrm{V} 1) \cdot 161 / \mathrm{m},
$$

where $\mathrm{C}_{\mathrm{NaOH}}$ is $\mathrm{NaOH}$ concentration, mol/l; $\mathrm{V}_{1}$ and $\mathrm{V}_{2}$ is added volumes of $\mathrm{NaOH}(\Delta \mathrm{V}), \mathrm{l} ; 161 \mathrm{~g} / \mathrm{mol}$ is molar weight of GlcN unit $\left(\mathrm{C}_{6} \mathrm{H}_{11} \mathrm{O}_{4} \mathrm{~N}\right), \mathrm{m}$ is the mass of the sample, $\mathrm{g}$.

\section{Statistics}

Statistical analysis was conducted using Microsoft Excel software. Correlation analysis was carried out by Pearson's statistics which was shown to be suitable for any sample size [20]. At sample volume equal to $n=3$, correlation significance $p>0.95$ is achieved at $r>0.997$ for two-tailed test as in our case [21].

\section{Results And Discussion}

\section{Determination of deacetylation degree (DD)}

DD is the main parameter which distinguishes chitin from chitosan. It is defined as a molar ratio of D-glucosamine and $\mathrm{N}$-acetyl-D-glucosamine units in the polymer structure. It affects basic physico-chemical properties of the polysaccharide including solubility in diluted organic acids [2]. Most studies comparing different methods for DD determination, use commercially available samples of chitosan with DD>70\% $[3,5,22]$. Chitosan with lower DD is well described in literature however the number of studies comparing such samples using different methods is limited [23-25]. To compare methods of DD estimation, samples A2 and A3 with low DD were obtained from chitosan A1 using mild conditions of acetylation preventing decrease in chitosan MW. Samples A1-A3 were analyzed by ${ }^{1} \mathrm{H}-\mathrm{NMR}$, conductance titration (CT) [3], and FDUV [5,16] (Figure 1).

${ }^{1} \mathrm{H}-\mathrm{NMR}$-spectrometry is one of the most frequently used methods to determine DD and is recommended by the American Standard Test Method to be used as a standard [2]. Degradation of high molecular weight chitosan samples before the analysis is able to increase the quality of NMR spectra and the accuracy of the results [26]. However, degradation is the additional step which may complicate the analysis. The method of degradation can also affect DD estimation and may require sample purification after hydrolysis. Taking into account these considerations a degradation step was avoided. Proton spectra of A1 and its reacetylated derivatives A2 and A3 are shown in (Figure1a). The most specific signals are the second proton of D-glucosamine unit (GlcN) (Figure 1) mark 1 and the acetic group proton of $\mathrm{N}$-acetylD-glucosamine (NAcGlcN) (Figure 1) mark 2. To determine DD on the basis of $1 \mathrm{H}-\mathrm{NMR}$ spectrum several calculation methods are used $[27,28]$. DD was calculated by two equations most often used. In the first case DD was obtained by calculation of ratio between proton integrals at 3.2 and $2.0 \mathrm{ppm}$ using equation 1 


\section{Organic and Medicinal Chemistry International Journal}

(Section 2.3). As an alternative, the equation taking into account the ratio of integrals of all glucopyranose protons signals (Figure 1) marks 3,1 to the protons of acetyl group of NAcGlcN (Figure 1) mark 2 was chosen equation 2 .

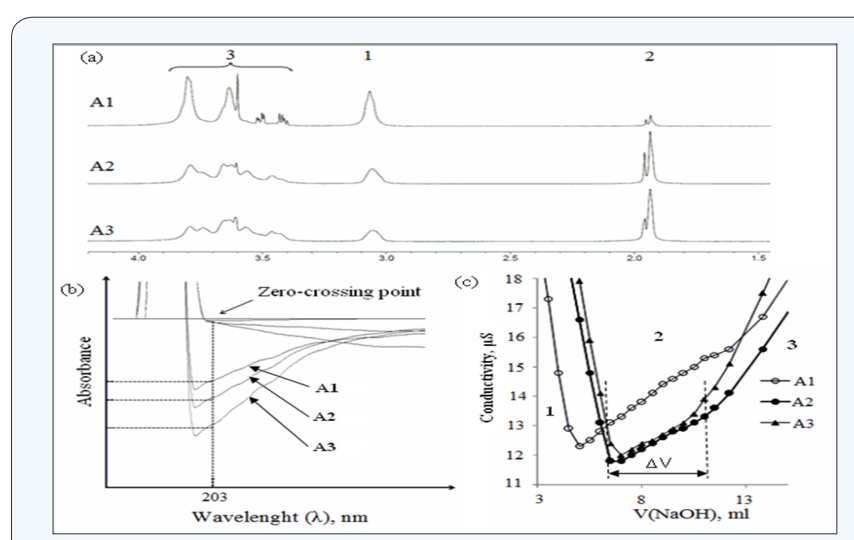

Figure 1: Determination of deacetylation degree (DD) of chitosan: a. 1H-NMR-spectra of chitosan samples A1-A3, numbers denote: 1 - acetic group of NAcGA proton signal; 2 signal of the second proton from GlcN; 3 - signal from protons in glucopyranose ring; b. first derivative of UV-absorption spectra obtained for chitosan samples A1-A3; c. conductometric titration curves for samples A1-A3, 1-3 relates to the regions on the titration curves (1- neutralization of unbound $\mathrm{HCl}$ in solution, 2neutralization of chitosan amino groups, 3- excess of hydroxide ions).

An alternative method for DD measurement is the analysis of the curves obtained by calculating the first derivative of UVabsorption spectra of NAcGlcN. This method is considered to be accurate, reproducible and does not demand high-skilled staff and expensive equipment [5]. FDUV curves are presented in (Figure 1b).

One more simple but accurate method to determine DD is conductometric titration (Figure 1c). There are also two methods to calculate DD on the basis of the titration curves equations 3-5.

The results on DD of chitosan A1-A3 obtained by different methods are shown in (Table 1). Absolute means of DD were close as determined by ${ }^{1} \mathrm{H}-\mathrm{NMR}(1)$, FDUV, and CT(1) while ${ }^{1} \mathrm{H}-\mathrm{NMR}(2)$ and CT(2) provided lower and higher values accordingly. It can be hypothesized that ${ }^{1} \mathrm{H}-\mathrm{NMR}(2)$ decreased estimation is a result of superimposition of glucopyranose protons signals while overestimation provided by CT(2) is a result of chitosan sedimentation in $\mathrm{NaOH}$ that impedes the detection of "plateau" on the titration curve (Figure 1).

\section{Correlation analysis of DD results}

For the analysis of the internal relation between chitosan samples, the data obtained by different methods were analyzed by Pearson's correlation statistics. For a small sample size $(n=3)$ non-parametric statistic is usually used. DD data analyzed by Spearman's rank correlation statistics provided $r= \pm 1$ for all methods used showing a high degree of correlation. More detailed analysis of DD data by parametric Pearson's correlation statistics demonstrated a high positive correlation reaching $95 \%$ confidence in most cases (Table 2). For example, the coefficient of correlation for CT (1) and CT (2) was $r=1$ in spite of the overestimated means provided by CT (2) calculation. The same high level of correlation was found between FDUV and CT (1). All other data gave slightly lower correlations which can possibly be explained by higher experimental errors. The estimated errors for $1 \mathrm{H}-\mathrm{NMR}(1), \mathrm{FDUV}$, and CT (1) are $10 \%, 2 \%$, and $3 \%$ accordingly. The reliable correlation of DD data as well as comparable DD values obtained by different methods show that any of the methods used determines chitosan DD rather accurately.

Table 2: Pearson's correlation coefficients between acetylation degree of chitosan samples A1-A3 determined by different methods.

\begin{tabular}{|c|c|c|c|c|c|}
\hline \multicolumn{6}{|c|}{ Deacetylation Degree (DD) } \\
\hline & ${ }^{1} \mathrm{H}-\mathrm{NMR}(1)^{\mathrm{a}}$ & ${ }^{1} \mathrm{H}-\mathrm{NMR}(2)^{\mathrm{b}}$ & FDUV $^{c}$ & $\mathrm{CT}(1)^{\mathrm{d}}$ & $\mathrm{CT}(2)^{\mathrm{e}}$ \\
\hline A1 & 90 & 94 & 91 & 96 & 118 \\
\hline A2 & 68 & 50 & 82 & 72 & 96 \\
\hline A3 & 56 & 43 & 55 & 55 & 82 \\
\hline \multicolumn{6}{|c|}{ Correlation coefficients, $r$} \\
\hline${ }^{1} \mathrm{H}-\mathrm{NMR}^{\mathrm{a}}$ & & 0.986 & 0.981 & 0.992 & 0.995 \\
\hline${ }^{1} \mathrm{H}^{-\mathrm{NMR}}{ }^{\mathrm{b}}$ & & & 0.934 & 0.956 & 0.964 \\
\hline FDUV & & & & $0.998^{\mathrm{f}}$ & 0.995 \\
\hline CT (1) & & & & & 1.000 \\
\hline
\end{tabular}

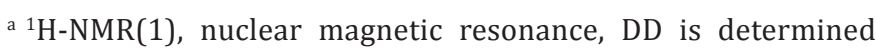
using equation (1).

b ${ }^{1} \mathrm{H}-\mathrm{NMR}(2)$, DD is determined using equation (2).

c FDUV, DD is determined using the first derivative of UVabsorbance spectra of NAcGlcN.

${ }^{\mathrm{d}} \mathrm{CT}(1)$, conductometric titration, DD is determined using equations $(3,4)$.

${ }^{\mathrm{e}} \mathrm{CT}(2)$, conductometric titration, DD is determined using equation (5).

fPearsons's correlation coefficients with probability $>95 \%$ ( $r>0.997)$ are shown in bold.

\section{Determination of chitosan molecular weight}

i. Viscometry: Molecular weight (MW) of chitosan is the second most important characteristic of the polymer that determines its physicochemical and biological properties. In spite of a great number of methods used to estimate MW, many of them are secondary as they calculate MW through hydrodynamic volume or gyration radius of the molecules which depend on the range of different factors (nature of solvent and its ionic strength, pressure, etc.). Many methods use standards: proteins, dextrans, polymer beads etc., which behavior in aqueous solutions is more predictable $[29,30]$. Charged polysaccharides including chitosan have a tendency 


\section{Organic and Medicinal Chemistry International Journal}

to aggregate in aqueous solutions which influences their hydrodynamic properties [7,31,32]. Methods of chitosan MW determination include viscometry, size exclusion chromatography (SEC), dynamic and static light scattering, etc. Among all, viscometry is one of the simplest. Intrinsic viscosity ( $\eta$ ) and MW can be related by Mark-Houwink equation: $[\eta]=\mathrm{kMW} \alpha$, where $\mathrm{k}$ and $\alpha$ are the empirically determined coefficients. Empirical constants $\mathrm{k}$ and $\alpha$ for chitosan vary significantly because of different conditions and methods used to obtain them [4,18,19,33-39] (Table 1). As a result there are no widely accepted coefficients for Mark-Houwink equation not only for chitosan, but also for other charged polymers.

Dynamic viscosity is another independent and widely used method characterizing chitosan and other polymers. For the analysis of samples considerably differing in viscosity, appropriate spindles are used. As with intrinsic viscosity, buffers and temperature are also the controlled parameters. The third standard method used to determine chitosan MW is SEC. SEC obtains MW in three forms: number average $\overline{M_{n}}$, weightaverage $\bar{M}_{w}$, and z-average $\bar{M}_{2}$; as well as it calculates dispersity index (DI). However there are limitations for SEC. Molecular SEC standards dextrans, pollutants, polyethylenoxide and others are uncharged polymers. Ci S.X. et al. as well as others showed that SEC profiles of uncharged polymers used as molecular SEC standards, are much narrower than of charged ones such as chitosan or alginate [30]. This corresponds to different physicochemical properties of charged and neutral polymers. SEC coupled with multi-angle light scattering (MALS) detector helps to overcome some obstacles related to the use of standards. SEC is also sensitive to the nature and ionic strength of the solvent as they affect hydrodynamic volume of the molecules [40].

Field flow fractionation (FFF) is a relatively new method for the analysis of chitosan behavior in aqueous solutions $[12,29]$. Contrary to SEC, fractionation by FFF occurs in flow at low pressure and in the absence of a solid phase. Coupling of FFF with MALS and refractometric detector gives a unique opportunity to determine $\mathrm{MW}$ in different chitosan fractions. On account of this, FFF finds a wide application for the analysis of different polymers including natural polysaccharides such as amylase and amyl pectin [41], cellulose [42], alginate [43] etc.

To compare MW determined by different methods, low MW chitosan samples B and C (DD>90\%) were prepared. Table 1 presents the experimental conditions for viscometry and the empirical coefficients of the Mark-Houwink equation taken from different published protocols. The results of viscometry experiments for samples A1-A3 are shown in (Table 3). Application of Mark-Houwink coefficients from protocol 1 to A1-A3 intrinsic viscosity data provided a significant increase in MW of samples with low DD, while both intrinsic and dynamic viscosity steadily decreased. Contrary to this, protocols 2 and 3 achieved decreasing MW with a decrease in DD, however the absolute MW values differed significantly ( $t$-test, $\mathrm{p}<0.01$ ). Note that the conditions used to obtain A2 and A3 were mild and MW was unchanged in contrast to alkaline deacetylation which induces partial hydrolysis of chitosan [44]. These results support earlier published observation that DD affects conformation of chitosan molecule leading to a different physicochemical behavior translated into different MW determined [45].

Table 3: Intrinsic and dynamic viscosities of chitosan samples A1-A3.

\begin{tabular}{|c|c|c|c|c|c|c|c|c|}
\hline & ${ }^{\mathrm{a} D D}$ & ${ }^{b}[\eta] d$, & ${ }^{c}[\eta](1)$ & ' $[\eta](2)$ & ${ }^{c}[\eta](3)$ & $\mathrm{d} M \eta(1)$ & $\mathrm{d} M \eta(2)$ & $\mathrm{d} \bar{M} \eta(3)$ \\
\hline & $\%$ & mPa.s & $\mathrm{ml} / \mathrm{g}$ & $\mathrm{ml} / \mathrm{g}$ & $\mathrm{ml} / \mathrm{g}$ & $\mathrm{kDa}$ & $\mathrm{kDa}$ & $\mathrm{kDa}$ \\
\hline A1 & $94 \pm 3$ & 7.4 & 740 & 730 & 710 & 770 & 300 & 204 \\
\hline A2 & $71 \pm 2$ & 5.3 & 590 & 590 & 540 & 1460 & 240 & 113 \\
\hline A3 & $55 \pm 1$ & 5.1 & 500 & 500 & 510 & 2600 & 220 & 109 \\
\hline
\end{tabular}

aDD, deacetylation degree was calculated as average as measured by 1H-NMR(1), FDUV, and CT(1) (Table 1).

${ }^{\mathrm{b}} \eta_{d}$ - dynamic viscosity.

${ }^{c} \eta(1), \eta(2), \eta(3)$ are intrinsic viscosities measured using protocols 1, 2, and 3 accordingly (Table 2).

$d \bar{M} \quad \eta(1), \bar{M} \eta(2), \bar{M} \eta(3)$ are molecular weights of Chi samples estimated by viscometry in protocols 1,2 , and 3 accordingly.
The most important observation was the stability of intrinsic viscosity of chitosan samples determined by different protocols ( $p>0.4$, t-test). It should be noted that protocol 3 , which used higher salt buffer, slightly decreased the means in comparison with protocols 1 and 2 which provided identical viscosity values. Analysis of samples A1, B, C with high DD demonstrated that characteristic viscosity did not vary much while absolute MW values determined using published Mark-Hauwink coefficients differed 3.7 to 28 times (Table 4). 


\section{Organic and Medicinal Chemistry International Journal}

Table 4: Correlation of molecular weight, intrinsic and dynamic viscosities of the highly deacetylated chitosan samples.

\begin{tabular}{|c|c|c|c|c|c|c|c|}
\hline & a $[\eta] d$ & ${ }^{b}[\eta](1)$ & ${ }^{\mathrm{b}}[\eta](2)$ & ${ }^{\mathrm{b}}[\eta](3)$ & ${ }^{c} \bar{M}_{\eta}(1)$ & ${ }^{c} \bar{M}_{\eta}(2)$ & ${ }^{\mathrm{c}} \overline{\mathbf{M}}_{\eta}(3)$ \\
\hline & m Pa.s & $\mathrm{ml} / \mathrm{g}$ & $\mathrm{ml} / \mathrm{g}$ & $\mathrm{ml} / \mathrm{g}$ & $\mathrm{kDa}$ & $\mathrm{kDa}$ & $\mathrm{kDa}$ \\
\hline $\mathrm{A} 1$ & 7.4 & 740 & 730 & 710 & 770 & 300 & 204 \\
\hline B & 1.1 & 90 & 110 & 100 & 76 & -35 & 13 \\
\hline C & 0.5 & 60 & 60 & 30 & 56 & 20 & 2 \\
\hline \multicolumn{8}{|c|}{ Pearsons's coefficients of correlation ( $r$ ) } \\
\hline$[\eta]_{d}$ & & 0.999 & 1.000 & 1.000 & 0.999 & 1.000 & 1.000 \\
\hline$[\eta](1)$ & & & 1.000 & 0.999 & 1.000 & 1.000 & 1.000 \\
\hline$[\eta](2)$ & & & & 1.000 & 0.999 & 1.000 & 1.000 \\
\hline$[\eta](3)$ & & & & & 0.998 & 0.999 & 0.999 \\
\hline $\bar{M} \eta(1)$ & & & & & & 1.000 & 1.000 \\
\hline $\bar{M} \eta(2)$ & & & & & & & 1.000 \\
\hline
\end{tabular}

a[n]d - dynamic viscosity.

${ }^{b} \eta(1), \eta(2), \eta(3)$ are intrinsic viscosities measured using protocols 1,2 , and 3 accordingly (Table 2 ).

${ }^{c} \bar{M}_{\eta}(1), \bar{M}_{\eta}(2), \bar{M}_{\eta}(3)$ are molecular weights of samples estimated by viscometry in protocols 1,2 , and 3 accordingly.

Statictically significant $(p<0.05)$ Pearsons's coefficients of correlation are shown in bold.

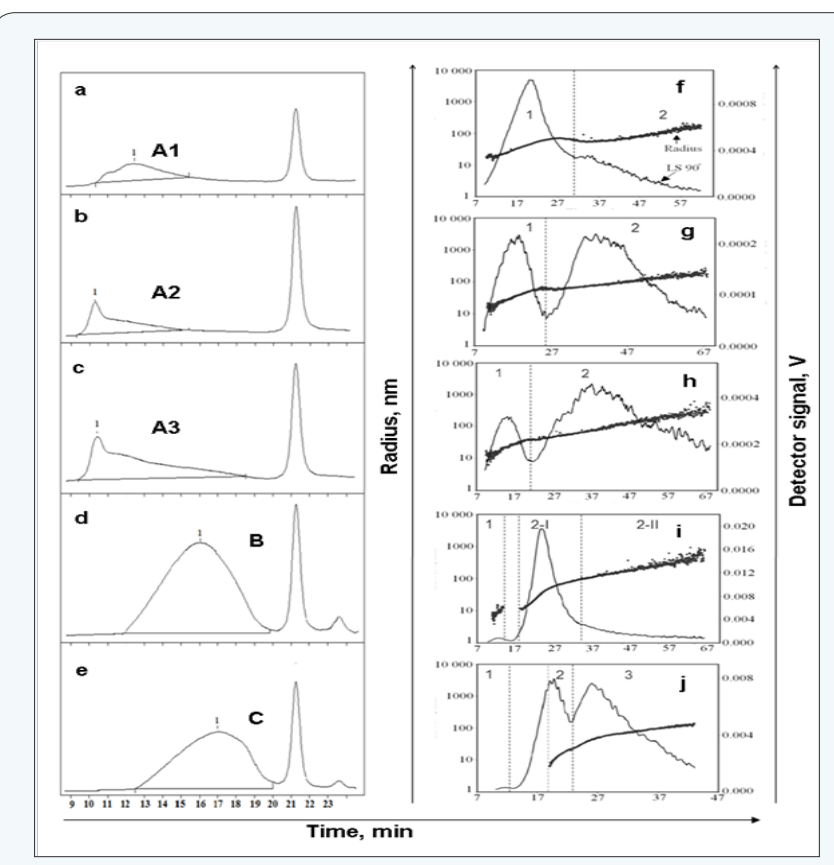

Figure 2: Size exclusion chromatography (a-e) and flow field fractionation ( $f-j)$ profiles of chitosan samples $A 1-A 3, B$, and C (from top to bottom): a-e. Note a significant asymmetry of A2 (b) and A3 (c) profiles associated with aggregation. 1 denotes the position of maximum of elution. $\mathrm{f}$-j. MALS signal is shown by histograms; radius of gyration corresponds to dotted curves. 1, 2, 2-I, 2-II, 3 denote peaks recognized by the system as different fractions. Molecular weights in Table 5 correspond to fraction 1. ii. Determination of chitosan MW using SEC and FFF: SEC and FFF elution profiles of chitosan samples A1-A3, B, and C are shown in (Figure 2) and the results are summarized in (Table 5). FFF data in (Table 5) are shown only for fraction 1 (Figure 2) which corresponds to non-aggregated chitosan. SEC failed to fractionate accurately low DD chitosans A2 and A3 (Table 5) possibly due to a massive chitosan aggregation which supports the results obtained by Schatz et al. [45]. Both SEC and FFF showed aggregation in samples A2 and A3 as revealed by asymmetric SEC profiles (Figure 2) and a significant increase in fraction 2 in FFF analysis (Figure 2).

Table 5: Molecular weights of chitosan samples determined by size exclusion chromatography (SEC), and field flow fractionation (FFF).

\begin{tabular}{|c|c|c|c|c|c|c|c|c|}
\hline & \multicolumn{3}{|c|}{ SEC, kDa } & $\begin{array}{c}{ }^{\mathrm{a} D I} \\
\text { SEC }\end{array}$ & \multicolumn{3}{c|}{${ }^{2}$ FFF, kDa } & $\begin{array}{c}{ }^{\mathrm{a}} \text { PI, } \\
\text { FFF }\end{array}$ \\
\hline & $\bar{M}_{\eta}$ & $\overline{\boldsymbol{M}}_{w}$ & $\overline{\boldsymbol{M}}_{\eta}$ & & $\bar{M}_{\eta}$ & $\bar{M}_{w}$ & $\bar{M}_{z}$ & \\
\hline A1 & 217 & 429 & 834 & 2.0 & 90 & 220 & 570 & 2.4 \\
\hline A2 & 430 & $>1000$ & $>3000$ & nd & 85 & 110 & 150 & 1.3 \\
\hline A3 & $>2000$ & $>2000$ & $>3000$ & nd & 47 & 62 & 82 & 1.3 \\
\hline B & 21 & 55 & 110 & 2.6 & 34 & 37 & 44 & 1.1 \\
\hline C & 13 & 36 & 70 & 2.8 & 26 & 27 & 29 & 1.0 \\
\hline
\end{tabular}

${ }^{a} \mathrm{DI}$ - dispersity index determined by SEC or FFF accordingly where $\mathrm{DI}=\bar{M} w / \bar{M} n$.

Aggregation of low DD chitosan samples was shown earlier by Popa-Nita et al. [13]. Fractionation of chitosan samples by FFF was undertaken to obtain chitosan samples with as low dispersity as possible. Indeed for low MW B and C chitosan samples dispersity index (DI) for fraction 1 in FFF was around 1 (Table 5) while for the same fraction 1 in A1 DI was found to be 


\section{Organic and Medicinal Chemistry International Journal}

even higher than as determined by SEC (2.4 vs 2.0). This result shows the intrinsic property of high molecular weight chitosan to form aggregates. Of note, aggregation of chitosan is evident for each sample (Figure 2) f-j, fractions 1, 2, 3.

\section{Correlation analysis of $\mathrm{MW}$ results}

Table 4 shows the results of Pearson's parametric correlation analysis of the viscosity data. In spite of a small number of samples correlation was significant $(\mathrm{p}<0.05)$. At the same time there was a high difference in MW, especially for the small molecule C (28 times). Pearson correlation coefficient is a measure of the linear distance between two variables $\mathrm{X}$ and $\mathrm{Y}$, giving a value between +1 and -1 , where 1 is total positive correlation, 0 is no correlation, and -1 is total negative correlation. It is widely used as a measure of the degree of linear dependence between two variables. Thus it means that the change in MW determined by different methods is highly proportional. At the same time intrinsic viscosities determined by different protocols showed very close results both in absolute values and correlation coefficients. High correlation between all viscosity data $(r>0.999, \mathrm{p}<0.05)$ (Table 4 ) shows that all three protocols recognize the difference in the size of chitosan molecules.

Table 6: Correlation between molecular weight data obtained by viscometry, size exclusion chromatography, and field flow fractionation.

\begin{tabular}{|c|c|c|c|c|c|c|c|c|c|}
\hline & \multicolumn{3}{|c|}{ Viscometry } & \multicolumn{3}{|c|}{ SEC } & \multicolumn{3}{|c|}{ FFF } \\
\hline & 1 & 2 & 3 & $\bar{M} n$ & $\bar{M} w$ & $\bar{M}_{z}$ & $\bar{M} n$ & $\bar{M} w$ & $\bar{M}_{z}$ \\
\hline A1 & 770 & 300 & 204 & 217 & 429 & 834 & 90 & 220 & 570 \\
\hline B & 76 & 35 & 13 & 21 & 55 & 110 & 34 & 37 & 44 \\
\hline $\mathrm{C}$ & 56 & 20 & 2 & 13 & 36 & 70 & 26 & 27 & 29 \\
\hline \multicolumn{10}{|c|}{ Pearson's coefficients of correlation, $r$} \\
\hline $\begin{array}{l}\text { Viscometry } \\
\text { protocol } 1\end{array}$ & & 1.00 & 1.00 & 1.00 & 1.00 & 1.00 & 0.996 & 1.00 & 1.00 \\
\hline $\begin{array}{l}\text { Viscometry } \\
\text { protocol } 2\end{array}$ & & & 1.00 & 1.00 & 1.00 & 1.00 & 0.998 & 1.00 & 1.00 \\
\hline $\begin{array}{l}\text { Viscometry } \\
\text { protocol } 3\end{array}$ & & & & 1.00 & 1.00 & 1.00 & 0.998 & 1.00 & 1.00 \\
\hline SEC $\bar{M} n$ & & & & & 1.00 & 1.00 & 0.997 & 1.00 & 1.00 \\
\hline $\operatorname{SEC} \bar{M} w$ & & & & & & 1.00 & 0.997 & 1.00 & 1.00 \\
\hline $\operatorname{SEC} \bar{M} z$ & & & & & & & 0.998 & 1.00 & 1.00 \\
\hline $\mathrm{FFF} \bar{M} n$ & & & & & & & & 0.998 & 0.996 \\
\hline $\operatorname{FFF} \bar{M} w$ & & & & & & & & & 1.00 \\
\hline
\end{tabular}

${ }^{a}$ All designations are as in Table 3.

The data on the comparison of MW measurements obtained by viscosity, SEC, and FFF for samples A1, B, and C are presented in (Table 6). The values of MW of each sample differed 2-8 times however a correlation between all data was highly significant $(p<0.05)$. High correlation of all data obtained by different methods demonstrates that all of them distinguish the difference between chitosan samples. However the absolute values of MW vary significantly possibly as a result of different physicochemical behavior of chitosan in different conditions.

Taken together it can be concluded that each method characterizes chitosan in a specific condition and none of them can produce the same MW values for the same sample, which is likely to mean than chitosan changes its conformation in different conditions and behaves as a molecule with different MW. The molar concentration of the buffers used for the viscometry studies increased in a row: $0.3<0.4<0.5 \mathrm{M}$ for protocols $1-3$ accordingly. MW of the same samples determined by these protocols decreased with the increase in molar concentration (Table 4). At the same time viscosity did not change much
(Table 4). Data on the decrease of both viscosity and MW in A1 after reacetylation (chitosan samples A2 and A3) support this conclusion. Viscometry, as the simplest and most direct method of chitosan analysis, should be preferred. Chitosan viscosity, either intrinsic or dynamic, should serve as a main chitosan characteristic.

Problems of MW determination are not unique for chitosan. Contrary to DNA and proteins, which have stable conformation, polysaccharides are prone to change it in different conditions (salt, temperature, pressure) and can easily form complexes [4650]. Our results demonstrate that MW may not be considered as the best characteristic of chitosan. Contrary to MW, viscosity is a much more stable characteristic of chitosan as well as of other polysaccharides.

\section{Conclusion}

The results of this paper demonstrated that deacetylation degree of chitosan molecules is a stable characteristic and can be determined sufficiently well by multiple modern methods. 


\section{Organic and Medicinal Chemistry International Journal}

Contrary to DD, we and many others showed that MW severely depends on the method of determination which usually is explained as a result of methodological problems: recalculation of MW from hydrodynamic volume or gyration radius using empirical coefficients; usage of neutral standards to obtain MW by SEC; different input of aggregation in some methods et cetera. At the same time, in spite of differences in the means of chitosan MW, Pearson's coefficients of correlations demonstrated high relation $(\mathrm{r}=1)$ between all the methods used $(\mathrm{p}<0.05)$ showing that all of them are sensitive to the changes in chitosan molecule mass or deacetylation degree. Taken collectively we have hypothesized that chitosan changes its conformation in different physicochemical conditions and behaves as a molecule with different MW. Contrary to many other methods, including highly advanced such as FFF, viscosity characterizes chitosan directly without recalculations and standards; it can be used for a wide range of chitosan molecules including samples with low MW and DD which are difficult for the analysis. We showed that viscosity data perfectly correlate with SEC and FFF data, while MW obtained by recalculation using Mark-Houwink coefficients, different MW standards, or gyration radius differ significantly. The uncertainty of chitosan MW determination is one of the main obstacles to its medicinal application. It can be suggested that the main characteristics of chitosan are DD and viscosity which completely and reliably identify the nature and predict the behavior of chitosan used for biomedical applications.

\section{Acknowledgement}

The research was supported by the Russian Scientific Foundation, grant №16-14-00046. We are grateful to Post nova Analytics Company (Germany) for FFF analysis of chitosan samples; to Dr. Valery Varlamov (Institute of Bioengineering, Moscow, Russia) for the fruitful discussion of the results; to Dr. Maxim Dubinnyi (IBCh, RAS, Moscow, Russia) for ${ }^{1} \mathrm{H}-\mathrm{NMR}$ studies.

\section{References}

1. K Richter (2011) Standardization of chitosan -first results of the inter lab study, Mater. 10-Th Int. Conf Eur Chitin Soc 4.

2. MR Kasaai (2009) Various methods for determination of the degree of N-acetylation of chitin and chitosan: A review. J Agric Food Chem 57(5): 1667-1676.

3. ES de Alvarenga, C Pereira de Oliveira, CR Bellato (2010) An approach to understanding the deacetylation degree of chitosan. Carbohydrate Polymers 80(4): 1155-1160.

4. ZM dos Santos, ALPF Caroni, MR Pereira, DR da Silva, JL Fonseca (2009) Determination of deacetylation degree of chitosan: a comparison between conductometric titration and $\mathrm{CHN}$ elemental analysis. Carbohydr Res 344(18): 2591-2595.

5. SC Tan, E Khor, TK Tan, SM Wong (1998) The degree of deacetylation of chitosan: Advocating the first derivative UV-spectrophotometry method of determination. Talanta 45(4): 713-719.

6. I V Blagodatskikh, EA Bezrodnykh, SS Abramchuk, A V Muranov, O V Sinitsyna et al. (2013) Short chain chitosan solutions: self-assembly and aggregates disruption effects. J Polym Res 20: 73.
7. E V Korchagina, OE Philippova (2010) Multichain aggregates in dilute solutions of associating polyelectrolyte keeping a constant size at the increase in the chain length of individual macromolecules. Biomacromolecules 11(2): 3457-3466.

8. G Lamarque, JM Lucas, C Viton, A Domard (2005) Physicochemical Behavior of Homogeneous Series of Acetylated Chitosans in Aqueous Solution: Role of Various Structural Parameters. Biomacromolecules 6(1): 131-142.

9. E Szymańska, K Winnicka (2015) Stability of chitosan-a challenge for pharmaceutical and biomedical applications. Mar Drugs 13(4): 18191846.

10. JC Giddings (1993) Field-flow fractionation: analysis of macromolecular, colloidal, and particulate materials. Science 260(5113): 1456-1465.

11.JC Giddings (1989) Field-flow fractionation of macromolecules. J Chromatogr 470(2): 327-335.

12. S Mao, C Augsten, K Mäder, T Kissel (2007) Characterization of chitosan and its derivatives using asymmetrical flow field-flow-fractionation: A comparison with traditional methods. J Pharm Biomed Anal 45(5): 736-741.

13. S Popa-Nita, P Alcouffe, C Rochas, L David, A Domard (2010) Continuum of structural organization from chitosan solutions to derived physical forms. Biomacromolecules 11(1): 6-12.

14. S Hirano, Y Kondo, K Fujii (1985) Preparation of acetylated derivatives of modified chito-oligosaccharides by the depolymerisation of partially $\mathrm{N}$-acetylated chitosan with nitrous acid. Carbohydr Res 144(2): 338341.

15. SV Rogozhin, AI Gamzazade, MA Chlenov, YeYu Leonova, et.al. (1988) The partial acidic hydrolysis of chitosan. Polym Sci U S S R 30(3): 607614.

16. RAA Muzzarelli, R Rocchetti (1985) Determination of the degree of acetilation of chitosans by first derivative ultravioletspectrophotometry. Carbohydrate Polymers 5(6): 461-472.

17.W Wang, SQ Bo, SQ Li, W Qin (1991) Determination of the MarkHouwink equation for chitosans with different degrees of deacetylation. Int J Biol Macro mol 13(5): 281-285.

18. M Rinaudo, M Milas, P Le Dung (1993) Characterization of chitosan Influence of ionic strength and degree of acetylation on chain expansion. Int J Biol Macromol 15(5): 281-285.

19. AI Gamzazade, VM Slimak, AM Skljar, E V Stykova, SSA Pavlova, et al (1985) Investigation of the hydrodynamic properties of chitosan solutions. Acta Polym 36(8): 420-424.

20. AK Gayen (1951) The frequency distribution of the product-moment correlation coefficient in random samples of any size drawn from nonnormal universes. Biometrika 38(1-2): 219-247.

21. RA Fisher, F Yates (1963) Statistical Tables for Biological, Agricultural and Medical Research, 6th ed. Longman Group Ltd. London 156.

22. R Czechowska-Biskup, D Jarosińska, B Rokita, P Ulański, JM Rosiak (2012) Determination of degree of deacetylation of chitosan comparison of methods, Prog Chemisrty Appl Chitin Its Deriv XVII: $5-20$.

23. T Kiang, J Wen, HW Lim, KW Leong (2004) The effect of the degree of chitosan deacetylation on the efficiency of gene transfection. Biomaterials 25(22): 5293-5301.

24.H Zhou, X Chen, M Kong, C Liu, et al. (2008) Effect of molecular weight and degree of chitosan deacetylation on the preparation and characteristics of chitosan thermo sensitive hydrogel as a delivery system. Carbohydrate Polymers 73(2): 265-273. 


\section{Organic and Medicinal Chemistry International Journal}

25. KM Vårum, MW Antohonsen, H Grasdalen, O Smidsrød (1991) Determination of the degree of $\mathrm{N}$-acetylation and the distribution of $\mathrm{N}$-acetyl groups in partially $\mathrm{N}$-deacetylated chitins (chitosans) by highfield n.m.r. spectroscopy. Carbohydr Res 211(1): 17-23.

26. A Hirai, H Odani, A Nakajima (1991) Determination of degree of deacetylation of chitosan by 1 H NMR spectroscopy. Polym Bull 26(1): 87-94.

27. M Lavertu, Z Xia, a N Serreqi, M Berrada, A Rodrigues, et al. (2003) A validated $1 \mathrm{H}$ NMR method for the determination of the degree of deacetylation of chitosan. J Pharm Biomed Anal32(6): 1149-1158.

28. C Augsten, K Mäder (2008) Characterizing molar mass distributions and molecule structures of different chitosans using asymmetrical flow field-flow fractionation combined with multi-angle light scattering. Int J Pharm 351(1-2) 23-30.

29. SX Ci, TH Huynh, LW Louie, A Yang, BJ Beals, et al. (1999) Molecular mass distribution of sodium alginate by high-performance sizeexclusion chromatography. J Chromatogr A 864(2): 199-210.

30. A Zubareva, T Shcherbinina, VP Varlamov, E Svirshchevskaya (2015) Intracellular sorting of differently charged chitosan derivatives and chitosan-based nanoparticles, Nanoscale 7(17): 7942-7952.

31. MW Anthonsen, KM Varum, AM Hermansson, O Smidsrød, DA Brandt (1994) Aggregates in acidic solutions of chitosans detected by static laser light scattering. Carbohydr Polym 25(1): 13-23.

32. MW Anthonsen, KM Vårum, O Smidsrød (1993) Solution properties of chitosans: conformation and chain stiffness of chitosans with different degrees of N-acetylation. Carbohydr Polym 22(3): 193-201.

33. LA Berkovich, GI Timofeyeva, MP Tsyurupa, VA Davankov (1980) Hydrodynamic and conformational parameters of chitosane. Polym Sci U S S R 22(8): 2009-2018.

34. G Berth, H Dautzenberg, MG Peter (1998) Physico-chemical characterization of chitosans varying in degree of acetylation. Carbohydr Polym 36(2-3) 205-216.

35. N Errington, SE Harding, KM Varum, L Illum (1993) Hydrodynamic characterization of chitosans varying in degree of acetylation. Int J Biol Macromol 15(2): 113-117.

36. MR Kasaai, J Arul, G Charlet (2000) Intrinsic Viscosity - Molecular Weight Relationship for Chitosan. J Polym Sci Part B Polym Phisics 38(19): 2591-2598

37. GAF Roberts, JG Domszy (1982) Determination of the viscometric constants for chitosan. Int J Biol Macromol 4(6): 374-377.

38. C Yomota, T Miyazaki, S Okada (1993) Determination of the viscometric constants for chitosan and the application of universal calibration procedure in its gel permeation chromatography. Colloid Polym Sci 271(10): 76-82.

39. SA Lopatin, MS Derbeneva, SN Kulikov, VP Varlamov, OA Shpigun (2009) Fractionation of chitosan by ultrafiltration. J Anal Chem 64(6):648-651.

40. E Chiaramonte, L Rhazi, T Aussenac, DR White (2012) Amylose and amylopectin in starch by asymmetric flow field-flow fractionation with multi-angle light scattering and refractive index detection (AF4-MALSRI). J Cereal Sci 56(2): 457-463.

41. B Wittgren, KG Wahlund (2000) Size characterization of modified celluloses in various solvents using flow FFF-MALS and MB-MALS. Carbohydr Polym 43(1): 63-73.

42. G Yohannes, M Jussila, K Hartonen, ML Riekkola (2011) Asymmetrical flow field-flow fractionation technique for separation and characterization of biopolymers and bioparticles. J Chromatogr A 1218(27): 4104-4116.

43. KLB Chang, G Tsai, J Lee, W-R Fu (1997) Heterogeneous N-deacetilation of chitin in alkaline solution. Carbohydr Reseach 303(3): 327-332.

44. C Schatz, C Viton, T Delair, C Pichot, A Domard (2003) Typical physicochemical behaviors of chitosan in aqueous solution. Biomacromolecules 4(3): 641-648.

45. SE Harding, KM Vårum, BT Stokke, O Smidsrod (1991) Molecular weight determination of polysaccharides, in: Adv. Carbohydrate Analysis. JAI Press Birmingham pp: 63-144.

46. L Piculell, J Borgström, I Chronakis, P-O Quist, C Viebke (1997) Organisation and association of $\kappa$-carrageenan helices under different salt conditions. Int J Biol Macromol 21(1-2): 141-153.

47. J Kang, X Hua, R Yang, Y Chen, H Yang (2015) Characterization of natural low-methoxyl pectin from sunflower head extracted by sodium citrate and purified by ultrafiltration. Food Chem 180: 98-105

48.X Li, S Al-Assaf, Y Fang, G0 Phillips (2013) Characterisation of commercial LM-pectin in aqueous solution. Carbohydr Polym 92(2): 1133-1142.

49. B Kim, S Woo, YS Park, E Hwang, MH Moon (2015) Ionic strength effect on molecular structure of hyaluronic acid investigated by flow fieldflow fractionation and multiangle light scattering. Anal Bioanal Chem 407(5): 1327-1334

50. AMF Lima, V Soldi, R Borsali (2009) Dynamic light scattering and viscosimetry of aqueous solutions of pectin, sodium alginate and their mixtures: effects of added salt, concentration, counterions, temperature and chelating agent. J Braz Chem Soc 20(9).

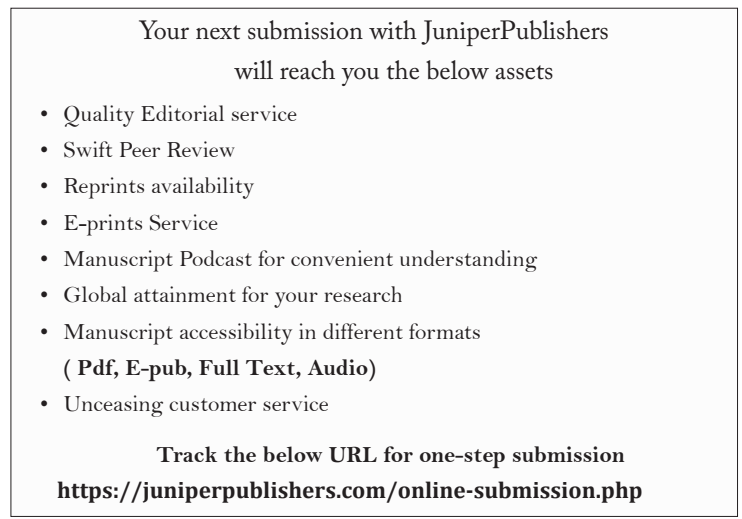

